\title{
Satisfaction, Preference and Injection Habits of Switching to 200 Units/ml Insulin Lispro Pen from 100 Units/ml: A Patient Survey in Germany
}

\author{
Jieling Chen · Paul Williams (D) · Magaly Perez-Nieves • \\ Carolina Piras De Oliveira · Erik Spaepen - Beatrice Osumili • \\ Jiat-Ling Poon · Nanette C. Schloot · Barbara Thun · Matthias Blüher
}

Received: June 23, 2021 / Accepted: September 2, 2021 / Published online: September 20, 2021

(C) The Author(s) 2021

\section{ABSTRACT}

Introduction: The study was designed to assess patient satisfaction, preferences and injection

Supplementary Information The online version contains supplementary material available at https:// doi.org/10.1007/s13300-021-01150-7.

J. Chen · M. Perez-Nieves · C. Piras De Oliveira • J.-L. Poon

Lilly Corporate Center, Indianapolis, IN 46285, USA

P. Williams $(\bowtie)$

IQVIA, Real-World Solutions, 17 bis place des

Reflets, Tour D2, TSA 64567, 92099 La Defense

Cedex, France

e-mail: paul.williams@iqvia.com

E. Spaepen

HaaPACS GmbH, Bahnhofstraße 19C, 69198

Schriesheim, Germany

B. Osumili

Eli Lilly and Company Limited, 8 Arlington Square

West, Downshire Way, Bracknell RG12 1PU, UK

N. C. Schloot

Lilly Deutschland GmbH, Werner-Reimers-Str. 2, 61352 Bad Homburg vor der Höhe, Germany

B. Thun

IQVIA Commercial GmbH \& Co. OHG, Real-World Solutions, Landshuter Allee 10, 80637 München, Germany

\section{Blüher}

Klinik und Poliklinik für Endokrinologie,

Nephrologie, Rheumatologie, Universitätsklinikum Leipzig, Liebigstr. 20, 04103 Leipzig, Germany habits for patients using insulin lispro 200 units/ml pen (IL200) compared to their previously used disposable 100 units/ml mealtime insulin pen ("MTI-100 pen") in Germany. Methods: A site-based, cross-sectional study involving a self-reported survey and medical record extraction in patients with diabetes currently using IL200 for between 3 and 12 months and had previously used any disposable MTI100 pen.

Results: Of 114 patients included, 83.3\% were satisfied with IL200 and 3.5\% were dissatisfied; $70.2 \%$ preferred IL200 over their previous MTI100 pen and $4.4 \%$ preferred their previous MTI100 pen. The main reasons for IL200 preference were the amount of insulin the pen carries, longer use before discarding, number of nonempty pens discarded, injection volume and frequency replacing pens. Patients discarded (median) 4 IL200 pens per month with 5.3\% discarding more than 10 units in their last pen. When insufficient insulin remained to complete a dose, $74.6 \%$ injected the remainder and completed with a new pen, $19.3 \%$ discarded the pen with remaining insulin, $7.0 \%$ saved it for future use and $1.8 \%$ left the dose incomplete.

Conclusions: Satisfaction and preference for IL200 was high in this sample of patients using IL200 for 3-12 months. Reasons were consistent with IL200 features, explaining the better patient experience and potential resource saving transitioning from a disposable MTI-100 pen. 
Keywords: Concentrated insulin; Diabetes mellitus; Insulin lispro 200 units/ml; Mealtime insulin; Patient preference; Patient satisfaction; Patient survey

\section{Key Summary Points}

Why carry out this study?

Concentrated insulin was developed to improve insulin therapy experience but little is known to what extent patients perceive an improved experience and why.

This study assessed patient satisfaction and preferences for IL200 in comparison to previous disposable 100 units/ml mealtime insulin pens through a realworld survey conducted in Germany.

\section{What was learned from the study?}

$83.3 \%$ of patients reported being satisfied with their current IL200 treatment.

The most common attributes that patients preferred for IL200 were the amount of insulin it contains, longer use, number of non-empty pens discarded, injection volume and frequency replacing pens; whereas no attribute was more commonly preferred for MTI-100.

\section{INTRODUCTION}

In 2019 it was estimated that approximately $9.3 \%$ of adults aged $20-79$ years worldwide had diabetes mellitus (DM), with numbers continuing to increase [1]. Germany has the one of the highest prevalences of DM worldwide with approximately $15.3 \%$ of the adult population [2]. Persons with type 1 DM (T1DM) are primarily treated by insulin therapy whereas persons with uncontrolled type 2 DM (T2DM) commonly use insulin after failing oral antidiabetic agents, diet and exercise therapy [3]. As a consequence of increased body weight and insulin resistance, persons with diabetes are challenged by a necessity for ever-increasing insulin doses [4-6] resulting in increased number of injections and higher injection volumes in order to maintain adequate glycaemic control $[7,8]$.

Concentrated insulin, which allows injection of the same amount of insulin in a smaller injection volume, may permit fewer injections, decreased injection site discomfort, and less frequent insulin pen changes, thus potentially less insulin wastage and/or fewer split doses $[9,10]$. It has also been demonstrated that the smaller injection volume results in lower glide force associated with easier insulin delivery [5]. Insulin lispro 200 units/ml (IL200) is the only concentrated bolus insulin and is known in Germany as Humalog ${ }^{\circledR} 200$ units/ml KwikPen $^{\mathrm{TM}}$ or Liprolog ${ }^{\circledR} 200$ units/ml KwikPen ${ }^{\mathrm{TM}}$. The IL200 pen contains total 600 units of insulin $(3 \mathrm{~mL}$ of 200 units $/ \mathrm{ml})$ with minimum dose of 1 unit, maximum single dose of 60 units, and with single unit increments. IL200 contains half of the injection volume of the bioequivalent Humalog $^{\circledR} 100$ units/ml KwikPen $^{\mathrm{TM}}$ (IL100). No extra adjustment is needed when moving from 100 units/ml insulin to IL200 [4, 5].

Greater satisfaction with insulin treatment has been associated with better compliance and improved persistence [11, 12], leading to improved glycaemic control [13]. Yet little is known about patient satisfaction and preference of IL200 and 100 units $/ \mathrm{ml}$ insulin in disposal pens. In a crossover simulated-use preference study, Wang et al. demonstrated that most patients or their caregivers preferred the IL200 pen ( $n=80,79.2 \%)$ compared to IL100 or reported no preference [14]. Key attributes that influenced IL200 preference included injection volume, increased amount of insulin units per pen and ease of pressing the injection button [14]. As satisfaction and preference in a simulated-use setting may not accurately reflect reallife experiences on routine use of IL200, a realworld study was required. Accordingly, the study presented here was designed to assess patient satisfaction and preferences for IL200 in comparison to previous disposable $100 \mathrm{units} / \mathrm{ml}$ mealtime insulin pens of any brand ("MTI-100 pen") in Germany. 


\section{METHODS}

\section{Patient Survey Development}

As an initial step, a study-specific survey was developed in order to comprehensively capture satisfaction and attributes of disposable insulin pens. To understand concepts relevant to persons with diabetes using prefilled MTI pens, this development process consisted of a literature review to identify concepts related to MTI pen satisfaction or preference from previous studies or surveys, four interviews with healthcare providers in Germany and four focus groups of persons with diabetes $(n=32)$. A total of 62 concepts were identified which were further reduced for relevance and conciseness to create a practical survey without excessive respondent burden. The remaining concepts were categorized into five themes: 'Device characteristics', 'Dose administration', 'Mealtime insulin dosing behaviours', 'Impact of device on lifestyle', and 'Satisfaction with treatment and injecting device'. From these concepts, survey items were created by distinguishing general treatment satisfaction (1 overall item and 5 sub-concept items) from patient preferences captured by specific device attributes ( 1 overall item and 23 attributes). Attributes were written in simple neutral statements with consistent equidistant response scales for both IL200 and MTI-100. To minimise possible bias towards IL200, respondents were allowed to choose the degree of preference (slight or strong) or neutrality. Where possible, items included opt-out responses to minimize missing data. The draft survey was reviewed by five key opinion leaders familiar with the MTI pens, including one physician in Germany (Professor Blüher). The draft survey was further cognitively debriefed in a sample of five persons with diabetes using disposable MTI pens in the USA before translating into German by native German speakers. The resulting draft survey consisted of screening questions, sociodemographic and clinical information questions, patient satisfaction items and preferences items.

The final step in the survey development was a pilot study in a sample of 40 persons with diabetes in Germany where comprehension of the survey was further assessed via usability questions. The final survey can be reviewed in the supplemental materials.

\section{Study Methods}

This study was a site-based cross-sectional observational study in Germany. Physicians invited were among the highest IL200 pen prescribers in Germany based on an IQVIA prescription database covering $85 \%$ of all prescriptions filled in Germany. Participating physicians were responsible for patient recruitment, survey implementation and collection, and clinical data extraction from medical records.

Eligible patients were 18 years of age or older, diagnosed with either T1DM or T2DM, had previously used any disposable MTI-100 pen for at least 3 months and subsequently switched to the IL200 pen for at least 3 months and no more than 12 months. These durations were chosen to maximize recall of past MTI-100 usage whilst ensuring sufficient time of using IL200 to report on experiences. Patients also had to be on both basal and bolus insulin and had data available in their medical records for a minimum of 3 months prior to the switch from MTI-100 to IL200.

Patients were excluded if they had an insulin pump or vial and syringe as the mode of administration of their MTI for a minimum of 3 months prior to the switch date and for the entire treatment period of IL200, use of any other MTI (including human insulin or premixture analogues), or if they had participated in a diabetes-related clinical trial while using MTI within the last 2 years prior to study inclusion.

At the time of study inclusion, patients were asked to complete the survey in paper format unattended while at the study site, after having provided their informed consent. Patients were not contacted afterwards for any query clarifications. 


\section{Sample Size}

As a result of the lack of previous data about patient satisfaction of IL200 in Germany, the sample size for this study was calculated on the basis of statistical and feasibility criteria. A sample size of 190 patients was deemed reasonable to describe responses to the survey items on patient satisfaction and preference, assuming $10 \%$ missing responses to items. This sample size would yield precision of continuous variables of 0.15 standard deviations (SDs) with a $95 \%$ confidence interval.

\section{Statistical Analysis}

All variables were analysed descriptively, including number and percentages of responses (with missing responses included in the calculation of percentages) or means and SDs. Variables capturing insulin injection habits, treatment satisfaction and preferences were also reported in medians and interquartile ranges (IQR, indicating the 25 th to the 75 th percentile of the distribution) when appropriate.

To examine the association between treatment satisfaction and patient's characteristics, univariable and multivariable proportional odds logistic regression analyses were performed. As a result of low frequency in dissatisfaction responses, treatment satisfaction was analysed as a three-category outcome instead of five: Very satisfied, Somewhat satisfied, Not satisfied (Neither satisfied nor dissatisfied + Somewhat dissatisfied + Very dissatisfied).

A comprehensive set of pre-selected variables for patient characteristics was explored. Age, gender, body mass index (BMI) at switch to IL200, IL200 dose at study inclusion and years on mealtime insulin were retained in the regression analysis regardless of $p$ value, either to control for effects thought to be important clinically but may not be apparent in a small sample or as potential confounding variables. The rest of the variables were explored using a stepwise selection approach: secondary school education, current employment outside home, Charlson comorbidity index (CCI), diabetes type, brand of MTI-100 prior to switch, HbA1c at study inclusion and at switch of $<7.0 \%$ $(<53 \mathrm{mmol} / \mathrm{mol}) \quad$ and $<8.0 \%$ ( $<64 \mathrm{mmol} / \mathrm{mol})$, concentrated basal insulin use during IL200 treatment, number of concomitant antidiabetic medications, and time since switch to IL200. Variables were retained if associated with a $p$ value $<0.15$.

Given the descriptive purpose of most of the study objectives, study variables were not imputed. The statistical analysis was conducted with statistical software SAS ${ }^{\circledR}$ version 7.13 (SAS Enterprise Guide).

\section{Compliance with Ethical Guidelines}

This study was conducted in accordance with the ethical principles that have their origin in the Declaration of Helsinki and that are consistent with Good Pharmacoepidemiology Practices (GPPs) and applicable laws and regulations of Germany where the study has been conducted. Approval was obtained from the Ethics Committee at the Saxon State Medical Association. A full list of the ethics committees is provided in Table S1 in the Supplementary Material. All patients provided informed consent to participate in the study.

\section{RESULTS}

\section{Study Population}

A total of 120 patients were included from 10 sites in Germany with a range of 2-21 recruited patients per site. The physicians of these sites consisted of 10 diabetologists, endocrinologists and/or internal medicine physicians with experience managing persons with diabetes in routine clinical practice. Six patients were excluded from the analysis as they did not meet the inclusion or exclusion criteria. This resulted in a study population of 114 patients, 12 with T1DM and 102 with T2DM.

The patient characteristics are described in Table 1 . The mean age was 63.2 years $(\mathrm{SD} \pm$ 12.9 ) at study inclusion, $45.6 \%$ of patients were female and $54.4 \%$ were male. BMI was available 
Table 1 General demographic and clinical characteristics

Overall $(n=114)$

Age at study inclusion

$n(\%)$

$114(100.0 \%)$

Mean (SD)

$63.2(12.9)$

Gender, $n(\%)$

Male

$62(54.4 \%)$

Female

Highest level of formal education completed at study inclusion, $n$ (\%)

Higher than secondary school degree

$67(58.8 \%)$

Secondary school degree or lower

$42(36.8 \%)$

Missing

$5(4.4 \%)$

Current employment status at study inclusion, $n$ (\%)

Working outside of home

$32(28.1 \%)$

Retired/working from home/home based due to other reasons

$79(69.3 \%)$

Missing

$3(2.6 \%)$

Self-reported weight $(\mathrm{kg})$ at study inclusion

$n(\%)$

$99(86.8 \%)$

Mean (SD)

$106.1(19.9)$

Self-reported BMI $\left(\mathrm{kg} / \mathrm{m}^{2}\right)$ at study inclusion

$n(\%)$

$99(86.8 \%)$

Mean (SD)

$36.0(6.5)$

Underweight $(<18.5)$

$0(0.0 \%)$

Normal weight $(18.5$ to $<25.0)$

$3(2.6 \%)$

Overweight $(25.0$ to $<30.0)$

$15(13.2 \%)$

Obesity class $1(30.0$ to $<35.0)$

$26(22.8 \%)$

Obesity class $2(35.0$ to $<40.0)$

$34(29.8 \%)$

Obesity class $3(40.0$ to $<45.0)$

$12(10.5 \%)$

Morbid obesity $(\geq 45.0)$

$9(7.9 \%)$

Missing

$15(13.2 \%)$

Type of diabetes mellitus at study inclusion, $n$ (\%)

Type 1

$12(10.5 \%)$

Type 2

$102(89.5 \%)$

HbAlc $<8.0 \%(<64 \mathrm{mmol} / \mathrm{mol})$ at study inclusion, $n$ (\%)

Yes

$75(65.8 \%)$ 
Table 1 continued

\begin{tabular}{ll}
\hline & Overall $(\mathbf{n}=\mathbf{1 1 4})$ \\
\hline No & $33(28.9 \%)$ \\
Missing & $6(5.3)$ \\
Time since DM diagnosis until switch (months) & \\
$n(\%)$ & $114(100 \%)$ \\
Mean (SD) & $16.4(9.6)$ \\
$<1$ year & $2(1.8 \%)$ \\
$1-5$ years & $10(8.8 \%)$ \\
$5-10$ years & $12(10.5 \%)$ \\
$>10$ years & $90(78.9 \%)$ \\
Time since switch to IL200 (months) at study inclusion & \\
$n(\%)$ & $114(100 \%)$ \\
Mean $(S D)$ & $7.4(3.0)$ \\
3 to $<6$ months & $41(36.0 \%)$ \\
6 to $<9$ months & $31(27.2 \%)$ \\
9 to $\leq 12$ months & $42(36.8 \%)$ \\
Charlson comorbidity index at switch date & \\
$n(\%)$ & $114(100 \%)$ \\
Mean $(S D)$ & $3.8(1.8)$ \\
Score $1-2$ & $29(25.4 \%)$ \\
Score $3-4$ & $44(38.6 \%)$ \\
Score $\geq 5$ & $41(36.0 \%)$ \\
\hline
\end{tabular}

for $86.8 \%$ of patients and of these, mean BMI was $36.0 \mathrm{~kg} / \mathrm{m}^{2}(\mathrm{SD} \pm 6.5) ; 2.6 \%$ had normal weight (BMI 18.5 to $<25.0 \mathrm{~kg} / \mathrm{m}^{2}$ ) and $13.2 \%$ were overweight (BMI 25 to $<30 \mathrm{~kg} / \mathrm{m}^{2}$ ). More than two-thirds $(71.0 \%)$ were obese.

Most patients $(58.8 \%)$ had a higher than secondary school degree and $36.8 \%$ had a secondary school degree or lower. More than twothirds of patients (69.3\%) were retired, working from home or were home based due to other reasons and $28.1 \%$ worked outside of home.

On average, patients had been using IL200 for 7.4 months $(S D \pm 3.0)$ prior to study inclusion. There was a balanced representation across the required 3-12-months period: 3 to $<6$ months, $36.0 \%$; 6 to $<9$ months, $27.2 \%$; 9 to $\leq 12$ months, $36.8 \%$. On average, patients had diabetes for 16.4 years $(\mathrm{SD} \pm 9.6)$, ranging from 0 to 49 years since diagnosis. Most patients (78.9\%) were diagnosed more than 10 years ago. Reported HbA1c was below $8.0 \%$ $(<64 \mathrm{mmol} / \mathrm{mol})$ for two-thirds of patients $(65.8 \%)$, above $8.0 \% \quad(\geq 64 \mathrm{mmol} / \mathrm{mol})$ for $28.9 \%$ of patients, and missing for $5.3 \%$ of patients.

The mean CCI score was $3.8(\mathrm{SD} \pm 1.8)$ with the following patient distribution: score 1-2, $25.4 \%$; score $3-4,38.6 \%$; score $\geq 5,36.0 \%$. 


\section{Treatment Regime Prior to and After IL200 Initiation}

Prior to the switch to IL200, patients had used their previous MTI-100 pen for a mean of 68.2 months ( $\mathrm{SD} \pm 72.4) \quad$ (approximately 5.7 years) (Table 2 ). The average daily dose of basal insulin was similar prior to and after switch to IL200 (36.9 vs 36.3 units), which accounted for around $40 \%$ of the total daily insulin dose. Concentrated basal insulin use was observed in $38.6 \%$ of patients prior to switch (36.8\% glargine 300 units $/ \mathrm{ml} ; 1.8 \%$ degludec 200 units $/ \mathrm{ml}$ ) and in $53.5 \%$ of patients after switch, with average daily dose of 44.7 vs 38.9 units respectively. The average daily dose of bolus insulin was 49.5 units prior to switch and 57.6 units after switch. On average, the total daily insulin dosages were 0.9 units $/ \mathrm{kg}$ $(\mathrm{SD} \pm 0.4)$ both before and after switching to IL200.

Concomitant antidiabetic medication use was similar prior to and after switch to IL200. Prior to the switch, metformin was used by $19.3 \%$ of patients and GLP-1 receptor agonists by $16.7 \%$ of patients. During the entire IL200 treatment, metformin was used by $21.1 \%$ of patients and GLP-1 receptor agonists by $17.5 \%$ of patients.

\section{Injection Habits Related to Remaining Insulin in Mostly Consumed (or Depleted) IL200 Pens}

Patients reported discarding a median of 4 IL200 pens (IQR 2-6) per month (Table 3). The median number of discarded pens with remaining insulin was 0 (IQR 0-3) per month. Just over half the patients $(53.5 \%)$ reported that no insulin had remained in their last discarded pen, a third (35.1\%) reported that between 1 and 10 units of insulin had remained in their last discarded pen and only six patients (5.3\%) reported that more than 10 units of insulin had remained in their last discarded pen. In case of insufficient amount of insulin to complete the dose, most patients reported that they generally inject the remaining insulin and complete the dose with a new pen (74.6\%). One-fifth (19.3\%) of patients reported generally discarding the pen with the remaining insulin and 7.0\% reported generally saving the pen for future use when a lower dose was needed. Only $1.8 \%$ of patients reported generally injecting the remaining insulin and leaving the dose incomplete.

\section{Patient Satisfaction with IL200}

Overall, over four-fifths (83.3\%) of patients reported being satisfied with their current IL200 treatment, including half of the patients $(51.8 \%)$ being very satisfied and one-third (31.6\%) being somewhat satisfied. While 7.0\% were neither satisfied nor dissatisfied, only $2.6 \%$ were somewhat dissatisfied and $0.9 \%$ were very dissatisfied (Fig. 1). For $6.1 \%$ of patients, the response was missing.

Almost all patients agreed (either agreed or strongly agreed) that they were confident they could correctly take their insulin as prescribed (96.4\%) and were confident in achieving their HbA1c treatment goals (88.6\%). Furthermore, most patients agreed (either agreed or strongly agreed) that they felt in control of their diabetes $(81.6 \%)$, that IL200 met their expectations $(84.3 \%)$, and that they would recommend IL200 to friends or family with diabetes who required MTI (84.2\%). Other general satisfaction items for IL200 are presented in Supplementary Fig. S1.

Considering all five general satisfaction statements as a whole, most patients agreed (either agreed or strongly agreed) to at least four of the five specific satisfaction questions (median 5, IQR 4-5). Neutrality to any of these statements (neither disagree nor agree) was reported by around $10 \%$ of patients, and disagreement or missing responses to any of these statements was rare $(<5 \%)$.

\section{Preference}

Overall, 70.2\% of patients preferred IL200 (either strongly or slightly) over their previous MTI-100 pen (Fig. 1). Half of the patients (52.6\%) strongly preferred IL200, 17.5\% slightly preferred IL200, $17.5 \%$ did not report a 
Table 2 Treatment regimen prior to and after switch based on patient medical record

Treatment prior to switch

Treatment after switch

Treatment duration (months)

$n(\%)$

Mean (SD)

Average daily dose basal insulin (units)

$n(\%)$

Mean (SD)

Ratio basal/total daily insulin units

$n(\%)$

$113(99.1 \%)$

Mean (SD)

$0.4(0.1)$

$114(100.0 \%)$

$0.4(0.1)$

Use of concentrated basal insulin, $n$ (\%)

Yes

$44(38.6 \%)$

No

Missing

$114(100.0 \%)$

$68.2(72.4)$

$114(100.0 \%)$

$36.9(18.5)$

$114(100.0 \%)$

$36.3(16.1)$

Average daily dose concentrated basal insulin (units)

$n(\%)$

$44(38.6 \%)$

Mean (SD)

Average daily dose bolus insulin (units)

$n(\%)$

$113(99.1 \%)$

Mean (SD)
$53(46.5 \%)$

$0(0.0 \%)$
$61(53.5 \%)$
$61(53.5 \%)$

$38.9(16.8)$

$114(100.0 \%)$

$57.6(34.3)$

$114(100.0 \%)$

93.8 (44.0)

$99(86.8 \%)$

$0.9(0.4)$

$24(21.1 \%)$

$20(17.5 \%)$

$13(11.4 \%)$

$3(2.6 \%)$

$1(0.9 \%)$

$13(11.4 \%)$ 
Table 3 Injection habits related to IL200 pens based on patient survey

Overall $(n=114)$

Numbers of IL200 pens discarded every month

$n(\%)$

$99(86.8 \%)$

Number of patients reporting 0 discarded IL200 pens

$18(15.8 \%)$

Median [IQR]

$4[2,6]$

Number of IL200 pens discarded with remaining insulin every month

$n(\%)$

$96(84.2 \%)$

Number of patients reporting 0 discarded IL200 pens with remaining insulin

$56(49.1 \%)$

Median [IQR]

$0[0,3]$

Unit of insulin remaining in last discarded IL200 pens, $n$ (\%)

0 units

$61(53.5 \%)$

$1-10$ units

$40(35.1 \%)$

11-20 units

$4(3.5 \%)$

21-30 units

$1(0.9 \%)$

31-40 units

$0(0.0 \%)$

More than 40 units

$1(0.9 \%)$

Missing

Action in case of insufficient insulin in the pen ${ }^{\mathrm{a}}, n$ (\%)

Discard the pen

Inject the insulin remaining and complete with a new pen

$85(74.6 \%)$

Inject the insulin remaining and leave the dose incomplete

$2(1.8 \%)$

Save the pen for future use

$8(7.0 \%)$

This has not occurred

$3(2.6 \%)$

a Patients were allowed to report multiple responses to this item

preference, $1.8 \%$ slightly preferred their previous MTI-100 and 2.6\% strongly preferred their previous MTI-100.

Specific attributes of disposable MTI pens that patients were shown to indicate preferences on are presented in Fig. 2. Attributes of MTI pens that most patients indicated preference for IL200 (either strongly or slightly) were "amount of insulin the pen carries" (71.0\%), "number of days using the pen before discarding it" (68.4\%), "number of non-empty pens discarded every month" (66.7\%), "amount of liquid (volume) each time you inject the medication" (64.0\%), and "frequency of replacing insulin pen" (63.1\%). In comparison, the percentage of patients reporting preference for their previous MTI-100 pen (either strongly or slightly) ranged from $0.9 \%$ to $5.3 \%$ across all attributes.

Considering all 23 attributes of disposable MTI pens that patients were shown, most patients preferred IL200 (either strongly or slightly) for at least 6 of the 23 attributes (median 11, IQR 6-15). In comparison, most 


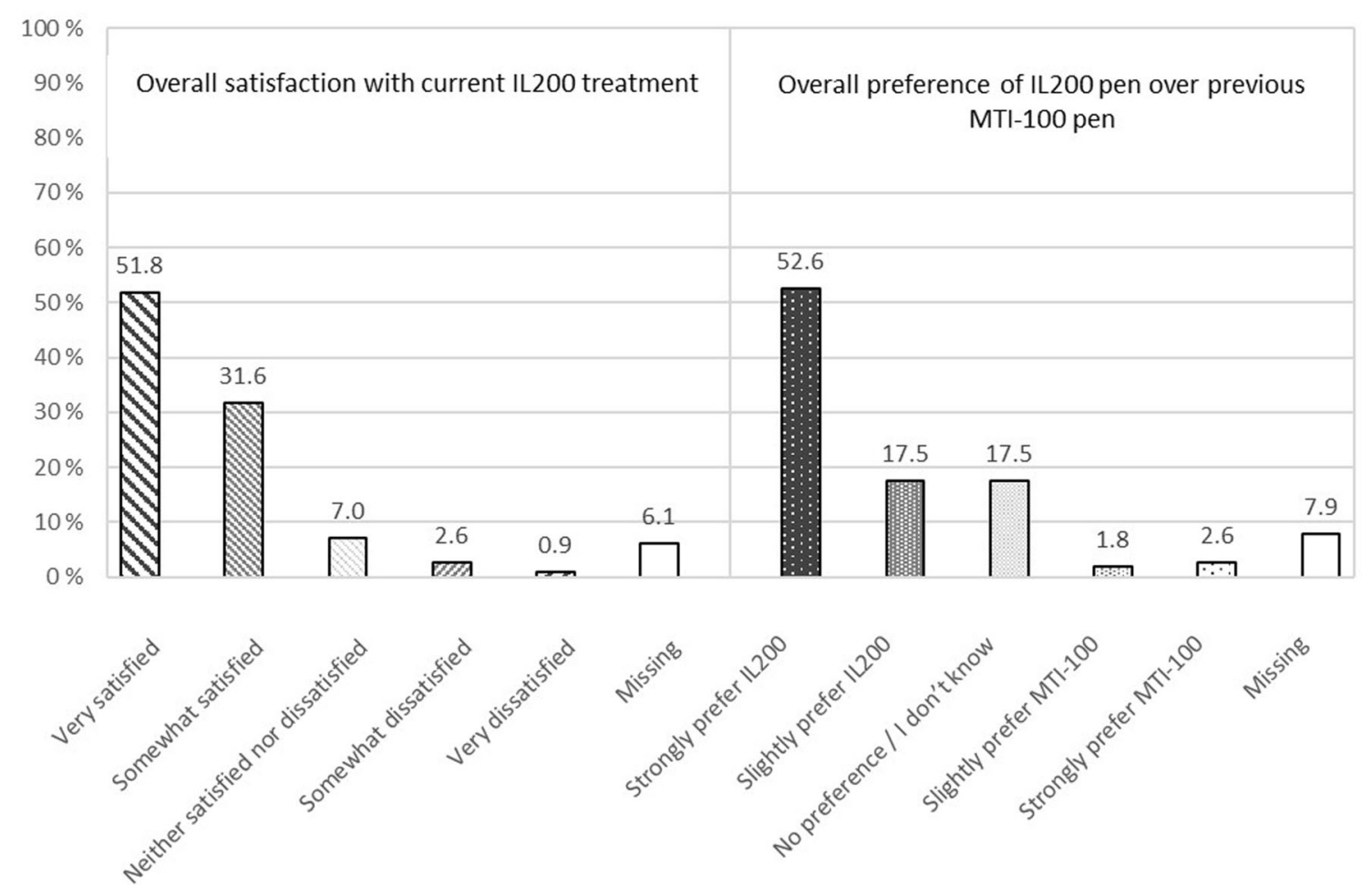

Fig. 1 Description of overall satisfaction and overall preference comparing IL200 with previous MTI-100 pen

patients did not prefer any attributes (neither strongly nor slightly) for their previous MTI-100 (median 0, IQR 0-0).

\section{Factors Associated with Satisfaction with IL200}

Higher satisfaction with IL200 was associated with more concomitant antidiabetic medications (odds ratio (OR) 2.70; $p=0.0019$ ), the time since switch between 6 and $<9$ months (OR $3.65 ; p=0.0272$ ) compared to 3 to $<6$ months, and HbA1c at study inclusion $<8.0 \% \quad(<64 \mathrm{mmol} / \mathrm{mol}) \quad(\mathrm{OR} \quad 3.14$; $p=0.0123) \quad$ compared $\quad$ to $\geq 8.0 \%$ ( $\geq 64 \mathrm{mmol} / \mathrm{mol}$ ). Age, gender, BMI at switch, secondary school education, IL200 dose at study inclusion and years on mealtime insulin were not significantly associated with IL200 satisfaction. Supplementary Table S2 presents the regression results examining factors associated with IL200 satisfaction.

\section{DISCUSSION}

In this sample of mostly elderly, retired, obese patients with T2DM who had been using IL200 for between 3 and 12 months, the vast majority $(83.3 \%)$ were satisfied with their IL200 treatment and $70.2 \%$ preferred it over their previous MTI-100. Only 3.5\% of patients reported being dissatisfied and only $4.4 \%$ reported preferring their previous MTI-100. This observation in our study of patients currently using IL200 is similar to the previous observation in experimental simulated-use settings where $79.2 \%$ of patients and caregivers expressed preference with IL200 compared to IL100 [14]. Although differences in study design and sample characteristics mean that the findings are not easily comparable, our study suggests that patient satisfaction for IL200 and preference for IL200 over their MTI-100 pens is also observed in real-world setting and thus suggests the benefits of IL200 exist in persons using it. 


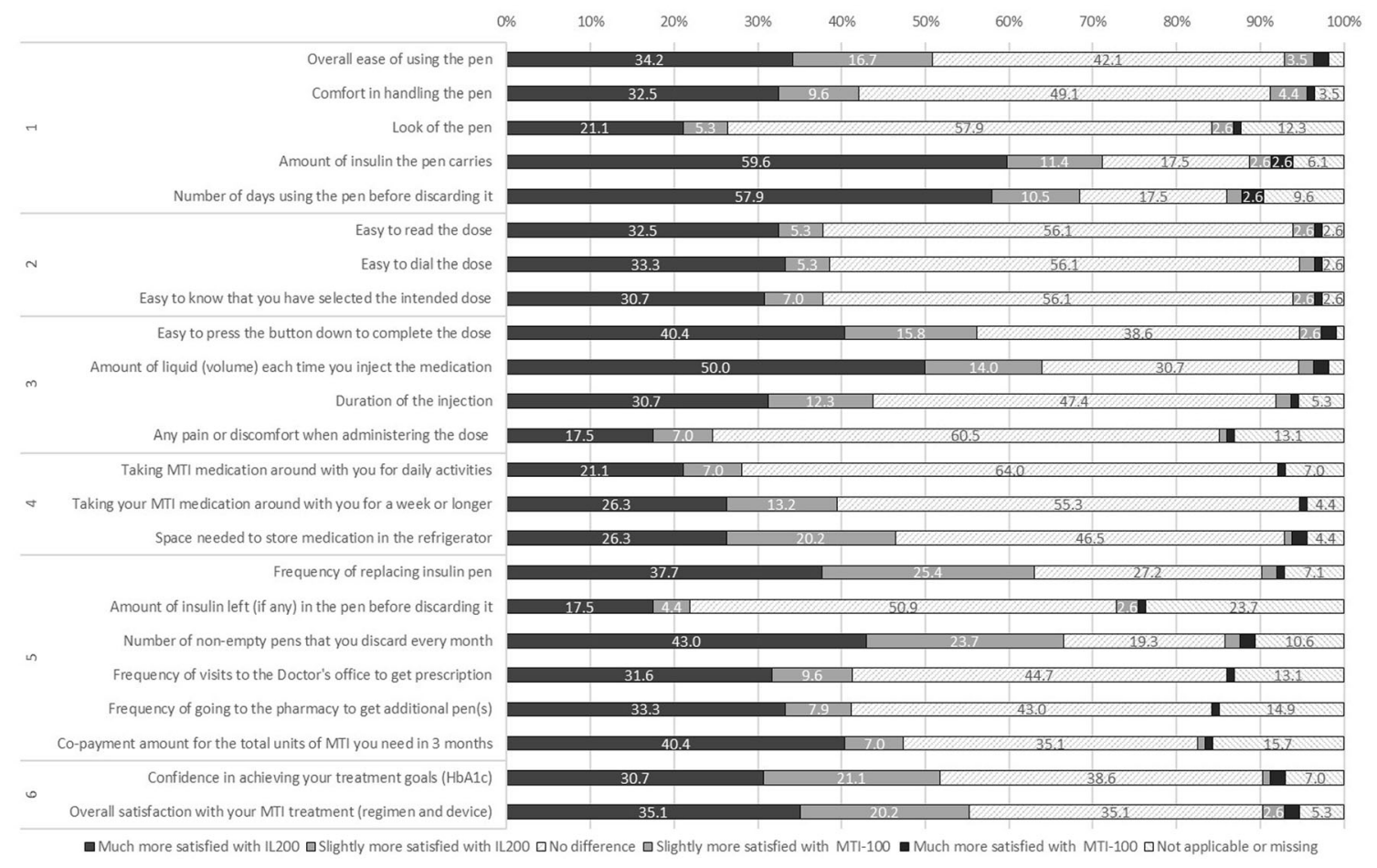

Fig. 2 Description of attributes capturing preference for current IL200 and previous MTI-100. Survey subcategories: $1=$ device characteristics, $2=$ dose preparation,

Similar observations of patient satisfaction were seen when patients were asked other treatment satisfaction questions in order to further understand their satisfaction for IL200. The vast majority of patients reported feeling confident they can take their insulin as prescribed, confident in achieving their treatment goals, felt in control of their diabetes, felt that IL200 met their expectations and would recommend IL200 pens to their friends or family with diabetes who require mealtime insulin.

Preference for IL200 was most notable concerning attributes of 'the higher amount of insulin that the IL200 pen carries' and the resulting 'ability of a longer use of the pen' along with 'a lower frequency of discarding it', the 'number of non-empty pens discarded every month' as well as the 'less volume needed to inject'. In context, of the median 4 IL200 pens discarded per month, the median number of pens with insulin remaining was 0 , indicating little wastage. This was also reflected by the majority of patients reporting 10 or fewer units
$3=$ dose administration, $4=$ impact on lifestyle, $5=\mathrm{im}$ pact on medication activities, $6=$ overall satisfaction. ${ }^{*}$ Percentages $<2.5 \%$ are not displayed

of insulin discarded in the last pen that they discarded. These observations are consistent with the intention of concentrated insulin pens such as IL200 to carry more units of insulin and thus lead to fewer pens being used and lower injection volume. Wang et al. (2016) observed in their simulated-use study that the top three reasons for IL200 preference included the total amount of insulin per pen and the amount of fluid injected [14] and our study suggests that patients also value strongly these attributes of IL200 after 3-12 months of IL200 use.

Attributes regarding the dose preparation, the look of the pen and the amount of insulin left in the pen before discarding it appeared of lesser relevance, although on the whole, patients preferred a median of 11 attributes for IL200 and a median of 0 attributes for their previous MTI-100.

Concerning 'the amount of insulin left in the pen before discarding it', it is important to consider the actions patients undertake when there is insufficient insulin in the pen to 
complete the dose. In our study, it was observed that most patients $(74.6 \%)$ reported injecting remaining insulin and completing the dose with a new pen. Although this study did not attempt to capture difference in pen use and insulin wastage between IL200 and MTI-100 pens, a previous study reported that patients using a MTI-100 pen discarded a mean of $5.5 \pm 8.2$ prefilled pens/cartridges monthly still containing insulin, each containing $8.6 \pm 8.7$ units of insulin, and that patients who discarded insulin considered it frustrating, time-consuming and painful to inject twice [10]. It is logical that a pen carrying more units of insulin should lead to fewer split-doses, which may explain why patient preferences were more apparent for attributes such as 'number of non-empty pens that you discard every month' compared to 'amount of insulin left in the pen when discarding it'. Similarly, in our study less than $2 \%$ of patients reported injecting the remaining insulin and leaving the dose incomplete, although this is considered as medically inadvisable. This percentage was lower than in another cross-sectional, self-reported survey of DM patients administering more than 20 units/day where $15 \%$ of patients reported of injecting an incomplete dose because of insufficient residual insulin in the pen [10]. Although we cannot draw comparisons between IL200 and MTI-100 injection behaviours, it is reasonable to assume that a pen containing more units of insulin leads to fewer occasions where there is opportunity to leave the dose incomplete because of insufficient insulin in the pen.

The proportional odds model investigating factors associated with IL200 satisfaction indicated that patients with more concomitant medications, a HbA1c at study inclusion of $<$ $8.0 \%(<64 \mathrm{mmol} / \mathrm{mol})$ and between 6 and 9 months of IL200 usage are more likely to be more satisfied with a pen that contains twice as many units of insulin and delivers the same insulin dose in half the volume. However, it should be noted that one of the main limitations of this analysis was the lack of variability in the satisfaction response to model the associations. As the majority of patients in our study were either highly satisfied or somewhat satisfied with IL200, consequently the regression model likely shows associations with highest satisfaction rather than throughout the entire range of possible satisfaction responses. Further research would be needed that includes a greater representation of patients dissatisfied with IL200 would be needed to help understand the relationships between any patient characteristics and IL200 satisfaction.

Concerning concomitant medications, it could be assumed that patients who already handle multiple medications might benefit more from the comfort of handling fewer pens and an easier handling due to the decreased volume. However, no evidence was found for a relationship between IL200 dose and satisfaction. HbA1c at study inclusion of $<8.0 \%$ $(<64 \mathrm{mmol} / \mathrm{mol})$ (i.e. a more controlled diabetes) was associated with an increase in the likelihood of reporting a higher satisfaction, which is likely influenced by the achievement of this treatment goal rather than by the current used MTI pen. Regarding the time since switch, likelihood of highest satisfaction was seen at 6 to $<9$ months which diminished at 9 to $\leq 12$ months. This may reflect increased satisfaction after the transition period, which can be challenging for patients [10], after which they are able to appreciate the benefits of a pen containing higher concentrated insulin and that satisfaction declines again when they get used to it and forget some of the practical disadvantages of MTI-100 treatment.

No evidence was found to suggest that any other demographic or clinical characteristic was associated with higher or lower satisfaction for IL200. It is possible that many physicians tend to prescribe IL200 to patients who are obese [15] because of higher insulin requirements of obese patients; however, our study did not support this in terms of differences in patient satisfaction for IL200 for different BMI groups.

Strengths of our study include the clinical confirmation of diagnosis data obtained by recruitment through physician and quality of data obtained through medical records extraction. In addition, the survey was developed through a robust process incorporating input from physicians and patients throughout. This helped to ensure that the survey was 
informative, comprehensive and easy to understand; and modifications were made iteratively to incorporate feedback received. Our study has some limitations. As a result of the real-world nature of the data and the descriptive purposes of most of the study objectives some study variables were missing and were not imputed. However, this also allowed patients who were unsure or preferred not to respond to do so which minimizes patients reporting inconsistently in order to complete the survey. A possible limitation of our study is that patients who may have been more dissatisfied may have already discontinued IL200 and would have been ineligible for our study; however, our findings appear in line with previous literature investigating preference between IL200 and IL100 in experimental settings, suggesting that this bias may not be so significant. Not all factors that may influence a prandial insulin choice were considered in our survey, although we assumed that this would not affect the patient's experience with IL200. The number of participants included in our study was lower than planned because of recruitment challenges and resulting in reduced precision in our estimates. Given the observed magnitude of reported satisfaction for IL200 and preference over previous MTI-100, it is improbable that substantially different findings would have been observed had our planned sample size been reached. We believe the lesser sample size impacted most the statistical power of our regression model. Finally, there was a lack of representation of T1DM, younger patients and non-obese patients, therefore concluding IL200 satisfaction and preferences for these groups are limited. However, our sample characteristics match closely to a recent retrospective cohort study of 811 IL200 users [15] which may lend confidence to the generalizability of our study and explain the underrepresentation of certain groups in our sample.

\section{CONCLUSION}

Overall, most patients who switched to IL200 from a previous MTI-100 pen reported being satisfied with IL200 and preferred IL200 pen over their previous MTI-100 pen. Consistent with the main differences between IL200 and other MTI-100 pens, preference for IL200 was mostly driven by the double amount of insulin contained in the same volume, ability of a longer use of the pen (number of days before discarding it) as well as the smaller volume needed to inject. This suggests a potential resource saving for payers and increased patients benefit from these product characteristics owing to fewer pens to store and to discard and lower frequency of replacing the pen, which may in turn lead to a better product experience for the patient, higher satisfaction and potentially better treatment adherence.

\section{ACKNOWLEDGEMENTS}

Funding. Sponsorship for this study and Rapid Service Fee were funded by Eli Lilly and Company, Indianapolis, USA.

Medical Writing, Editorial Assistance. Writing and editing support was provided by Annegret Gohlke from IQVIA. Support for this assistance was funded by Eli Lilly and Company.

Authorship. All named authors meet the International Committee of Medical Journal Editors (ICMJE) criteria for authorship for this article, take responsibility for the integrity of the work as a whole, and have given their approval for this version to be published.

Author Contribution. Jieling Chen, Paul Williams, Magaly Perez-Nieves, Carolina Piras De Oliveira, Beatrice Osumili, Nanette C. Schloot and Matthias Blüher made substantial contributions to the concept and design of the study. Jieling Chen, Paul Williams, Barbara Thun and Erik Spaepen analysed the data. Jieling Chen, Paul Williams, Magaly Perez-Nieves, Carolina Piras De Oliveira, Erik Spaepen, Beatrice Osumili, Jiat-Ling Poon, Nanette C. Schloot, Barbara Thun and Matthias Blüher provided interpretation of the data. Jieling 
Chen, Paul Williams and Barbara Thun drafted the manuscript.

Disclosures. Magaly Perez-Nieves, Carolina Piras De Oliveira, Beatrice Osumili, and JiatLing Poon are employees of Eli Lilly and Company. Magaly Perez-Nieves, and Jiat-Ling Poon are stockholders of Eli Lilly and Company. Jieling Chen was an employee and stockholder of Eli Lilly and Company and has no other conflicts to declare. Nanette C. Schloot is employee of Lilly Deutschland GmbH. Erik Spaepen received personal fees from Eli Lilly and Company. Paul Williams and Barbara Thun are employees of IQVIA, a vendor supporting Eli Lilly and Company for the study. Matthias Blüher reports personal fees from AstraZeneca, Amgen, Bayer, Boehringer-Ingelheim, DaiichiSankyo, Lilly, Novo Nordisk, Novartis and Sanofi.

Compliance with Ethical Guidelines. This study was conducted in accordance with the ethical principles that have their origin in the Declaration of Helsinki and that are consistent with Good Pharmacoepidemiology Practices (GPPs) and applicable laws and regulations of Germany where the study has been conducted. Approval was obtained from the Ethics Committee at the Saxon State Medical Association. A full list of the ethics committees is provided in Table S1 in the Supplementary Material. All patients provided informed consent to participate in the study.

Data Availability. The datasets generated during and/or analysed during the current study are available from the corresponding author on reasonable request.

Open Access. This article is licensed under a Creative Commons Attribution-NonCommercial 4.0 International License, which permits any non-commercial use, sharing, adaptation, distribution and reproduction in any medium or format, as long as you give appropriate credit to the original author(s) and the source, provide a link to the Creative Commons licence, and indicate if changes were made. The images or other third party material in this article are included in the article's Creative Commons licence, unless indicated otherwise in a credit line to the material. If material is not included in the article's Creative Commons licence and your intended use is not permitted by statutory regulation or exceeds the permitted use, you will need to obtain permission directly from the copyright holder. To view a copy of this licence, visit http://creativecommons.org/licenses/by$\mathrm{nc} / 4.0 /$.

\section{REFERENCES}

1. International Diabetes Federation. IDF Diabetes Atlas 9th Edition. 2019. https://www.diabetesatlas. org/en/. Accessed Jun 2, 2020.

2. International Diabetes Federation. IDF Europe members: Germany. https://www.idf.org/ournetwork/regions-members/europe/members/136germany.html. Accessed Nov 23, 2020.

3. Freeland B, Farber MS. A review of insulin for the treatment of diabetes mellitus. Home Healthc Now. 2016;34:416-23. https://doi.org/10.1097/NHH. 0000000000000446 .

4. Kalra S. High concentration insulin. Indian J Endocrinol Metab. 2018;22:160-3. https://doi.org/ 10.4103/ijem.IJEM_300_17.

5. Rees TM, Lennartz AH, Ignaut DA. A comparison of glide force characteristics between 2 prefilled insulin lispro pens. J Diabetes Sci Technol. 2015;9: 316-9. https://doi.org/10.1177/ 1932296814567533.

6. Watson L, Wilson BP, Alsop J, Kumar S. Weight and glycaemic control in type 2 diabetes: what is the outcome of insulin initiation? Diabetes Obes Metab. 2011;13:823-31. https://doi.org/10.1111/j. 1463-1326.2011.01413.x.

7. Inzucchi SE, Bergenstal RM, Buse JB, et al. Management of hyperglycemia in type 2 diabetes: a patient-centered approach: position statement of the American Diabetes Association (ADA) and the European Association for the Study of Diabetes (EASD). Diabetes Care. 2012;35:1364-79. https:// doi.org/10.2337/dc12-0413.

8. Kelly T, Yang W, Chen C-S, Reynolds K, He J. Global burden of obesity in 2005 and projections to 2030. Int J Obes (Lond). 2008;32:1431-7. https://doi.org/ 10.1038/ijo.2008.102. 
9. Schloot NC, Hood RC, Corrigan SM, Panek RL, Heise T. Concentrated insulins in current clinical practice. Diabetes Res Clin Pract. 2019;148:93-101. https://doi.org/10.1016/j.diabres.2018.12.007.

10. van Brunt K, Pedersini R, Rooney J, Corrigan SM. Behaviours, thoughts and perceptions around mealtime insulin usage and wastage among people with type 1 and type 2 diabetes mellitus: a crosssectional survey study. Diabetes Res Clin Pract. 2017;126:30-42. https://doi.org/10.1016/j.diabres. 2016.12.002.

11. Bakar ZA, Fahrni ML, Khan TM. Patient satisfaction and medication adherence assessment amongst patients at the diabetes medication therapy adherence clinic. Diabetes Metab Syndr. 2016;10: S139-43. https://doi.org/10.1016/j.dsx.2016.03. 015.

12. Emechebe N, Agu N, Malmi M, Zgibor JC. Examining the association between treatment satisfaction and medication adherence among patients with poorly controlled type 2 diabetes. Diabetes. 2018;67: 724-P. https://doi.org/10.2337/db18-724P.

13. Guerci B, Chanan N, Kaur S, Jasso-Mosqueda JG, Lew E. Lack of treatment persistence and treatment nonadherence as barriers to glycaemic control in patients with type 2 diabetes. Diabetes Ther. 2019;10:437-49. https://doi.org/10.1007/s13300019-0590-х.

14. Wang T, Conrad KA, van Brunt K, Rees TM. Attributes influencing insulin pen preference among caregivers and patients with diabetes who require greater than 20 units of mealtime insulin. J Diabetes Sci Technol. 2016;10:923-31. https://doi.org/ $10.1177 / 1932296816633232$.

15. Schloot NC, Perez-Nieves M, Sapin H, et al. Patient characteristics of insulin lispro 200 units/mL users in real world setting in Germany. Curr Med Res Opin. 2020;36:1611-7. https://doi.org/10.1080/ 03007995.2020.1815001. 